\title{
Mitigating Patient and Consumer Safety Risks When Using Conversational Assistants for Medical Information: Exploratory Mixed Methods Experiment
}

Timothy W Bickmore, PhD; Stefán Ólafsson, PhD; Teresa K O'Leary, BA

Khoury College of Computer Sciences, Northeastern University, Boston, MA, United States

\section{Corresponding Author:}

Timothy W Bickmore, $\mathrm{PhD}$

Khoury College of Computer Sciences

Northeastern University

360 Huntington Ave

524 ISEC

Boston, MA, 02115

United States

Phone: 16173735477

Email: t.bickmore@northeastern.edu

\section{Abstract}

Background: Prior studies have demonstrated the safety risks when patients and consumers use conversational assistants such as Apple's Siri and Amazon's Alexa for obtaining medical information.

Objective: The aim of this study is to evaluate two approaches to reducing the likelihood that patients or consumers will act on the potentially harmful medical information they receive from conversational assistants.

Methods: Participants were given medical problems to pose to conversational assistants that had been previously demonstrated to result in potentially harmful recommendations. Each conversational assistant's response was randomly varied to include either a correct or incorrect paraphrase of the query or a disclaimer message — or not - telling the participants that they should not act on the advice without first talking to a physician. The participants were then asked what actions they would take based on their interaction, along with the likelihood of taking the action. The reported actions were recorded and analyzed, and the participants were interviewed at the end of each interaction.

Results: A total of 32 participants completed the study, each interacting with 4 conversational assistants. The participants were on average aged 42.44 (SD 14.08) years, 53\% (17/32) were women, and 66\% (21/32) were college educated. Those participants who heard a correct paraphrase of their query were significantly more likely to state that they would follow the medical advice provided by the conversational assistant $\left(\chi_{1}^{2}=3.1 ; P=.04\right)$. Those participants who heard a disclaimer message were significantly more likely to say that they would contact a physician or health professional before acting on the medical advice received $\left(\chi_{1}^{2}=43.5\right.$; $P=.001)$.

Conclusions: Designers of conversational systems should consider incorporating both disclaimers and feedback on query understanding in response to user queries for medical advice. Unconstrained natural language input should not be used in systems designed specifically to provide medical advice.

(J Med Internet Res 2021;23(11):e30704) doi: 10.2196/30704

\section{KEYWORDS}

conversational assistant; conversational interface; dialogue system; medical error; patient safety; risk mitigation; warnings; disclaimers; grounding; explainability; mobile phone 


\section{Introduction}

\section{Background}

Conversational assistants (CAs) are general-purpose speech-based agents, such as Apple's Siri and Amazon's Alexa, that provide information or services through smartphones or smart speakers in the home. Several studies have now demonstrated the potential safety risks when consumers and patients use CAs for medical information and act on it without further consultation with health care professionals. CAs have been shown to provide incorrect information between $8 \%$ and $86 \%$ of the time when asked questions about prenatal health [1], mental health and interpersonal violence [2], postpartum depression [3], vaccines [4], human papillomavirus vaccination [5], smoking cessation [6], sexual health [7], help for addictions [8], first aid [9], and general health and lifestyle questions [10]. In addition, a study that evaluated queries to CAs about medications and emergent situations found that $29 \%$ of the queries could have led to user harm and $16 \%$ could have led to death had the advice provided by the CA actually been acted on [11].

Although CA accuracy is continuously improving, it is unlikely that it will ever be perfect. Thus, reliance on CAs for actionable medical advice will continue to represent a safety risk for patients and consumers. Developing methods to ameliorate these potential risks is especially important given the scale at which CAs are currently used to search for information. More than half $(56.4 \%)$ of the US adults use CAs on smartphones [12], and more than one-third (34.4\%) own 1 or more smart speakers with embedded CAs in their homes [13]. One-third of the 3.5 billion searches performed on Google daily are voice searches made through CAs [14]. A longitudinal study of smart speakers found that users gave a median 4.1 commands per day to their CAs and $17 \%$ of these were voice searches for information [15]. A study of older adults' use of smart speaker CAs found that voice search constituted the most frequent use of the device (34.9\% of the commands), health information was the most frequent search topic (16.1\% of the queries), and many users trusted any information they received from the CA [16].

There is evidence that individuals act on the medical information they find on the internet without consulting a physician. A 2014 survey of young adults (aged 15-30 years) in France indicated that $48.5 \%$ used the internet for health purposes, $33.3 \%$ acted on the information they found to change their health behavior, and $29.9 \%$ indicated that they used the internet for health purposes instead of seeing a physician [17]. A 2020 survey of Polish adults found that $76.8 \%$ used the internet for health information and $6.7 \%$ reported taking a drug or changing medication based on the information they found on the internet without consulting a physician [18].

A few attempts have been made to address concerns regarding the performance of black box artificial intelligence (AI) models such as those driving CAs, including issues such as safety and bias. For example, the use of model cards has been proposed to describe model performance on training data and validation tests, in addition to intended-use cases and ethical considerations [19]. Even with the high accuracies of state-of-the-art speech recognition and natural language understanding, errors still occur in the most ideal circumstances, and their prevalence increases in nonideal situations [20] or with users whose speech characteristics are underrepresented in the AI training data (eg, older adults, children, and nonnative speakers). Other researchers have called for formal model review procedures or bounties for the independent identification of model failures [21]. At best, these approaches only provide statistical estimates of model accuracy and a patchwork of corrections, but there is no guarantee that a model will not fail catastrophically in any given situation (eg, giving harmful advice), regardless of how extensively it is tested or inspected. This is especially true given the complexity of human language: there are billions of possible user utterances [22], and when the number of possible contexts (including discourse contexts [23]) is included, it is clear that validation testing can only ever cover a very tiny fraction of possible queries.

\section{Grounding in Communication}

A key concept in understanding errors in the interactive use of language is grounding. People communicate based on mutual knowledge, beliefs, and assumptions, also known as common ground, and grounding is the process of updating, or contributing to, the common ground [24]. Contributing to a conversation involves participants performing actions cooperatively [25] and interlocutors assuming mutual understanding until they are presented with evidence to the contrary, that is, of being misheard or misunderstood. For example, utterances such as "Huh?" and "What?" are commonplace verbal indicators of confusion in English.

Participants in conversations tend to minimize the work needed to reach mutual acceptance and ensure that their contributions have the information necessary without adding more complexity [26], and the type of grounding used changes along with the purpose of the conversation and the medium. Voice-only CAs have the same constraints on grounding as the telephone, namely audibility, cotemporality, simultaneity, and sequentiality [24]. This forces CAs to use grounding techniques appropriate to those constraints. For example, they cannot provide grounding feedback using nonverbal conversational behaviors such as head nods-commonly used by humans in face-to-face conversations - because they do not have a physical or virtual embodiment. Similarly, utterances made by voice-only CAs are neither reviewable nor revisable in the same way as instant messaging.

\section{Errors and Error Recovery in CA Interaction}

Several research efforts have reported on the kinds of errors that CAs make and the potential for recovering from them while interacting with users.

Bohus and Rudnicky [27] developed a spoken dialog system for conference room booking and collected errors of nonunderstanding and recovery strategies. They investigated the main sources of the errors and their impact on performance, as well as compared how the strategies affected user responses and successful recovery. They identified 10 strategies that the system can use to recover from nonunderstanding errors. The strategies that had the top 3 highest dialog recovery rates were 
as follows: (1) moving on to the next part of the task; (2) giving a full description of where they are in the dialog, what the problem is, and what the user can say at this point; and (3) telling the user what they can say at this point. Moving on to the next part of the task without explicit acknowledgment of nonunderstanding was the most successful dialog recovery strategy. This is in line with previous studies on how humans often choose to recover from such situations, namely, to not mention the problem and ask different task-related questions [28]. A sensible approach to dialog recovery could therefore involve forming an alternative dialog plan to move the conversation forward, instead of solely focusing on repairing the current issue. Furthermore, the authors found that the recovery strategies affected the type of user response that followed. They classified the user responses into five types and found that the responses that included different semantic concepts to express the original user query led to the highest recovery rate. Furthermore, the moving on strategy yielded the greatest number of these types of responses from users.

Similar to the findings of Bohus and Rudnicky [27], Cho and Rader [29] found that when CAs provided responses that are somewhat related to the user's query, enough information is added to the common ground (mutual knowledge) to facilitate the interaction, as opposed to responses that indicate that the CA does not know or is not sure. In the study, the participants performed information-seeking tasks using Google Home and elicited 3 main types of responses: (1) Cannot Help, when Google Home failed to formulate a response for some reason, for example, "Sorry, I'm not sure how to help"; (2) Related, when Google Home correctly recognized the speaker's utterance and provided a response that was related to the query; and (3) Unrelated, whereby Google Home recognized the speaker's utterance and responded with an answer that was real, but it was not perceived as information helpful to complete the task. Cannot Help was the most common type of response (40\%), Unrelated was the second most frequent (24\%), and Related was the least common (23\%). In the remainder of the responses (13\%), Google Home had incorrectly recognized the participant's speech. Utterances of the Cannot Help variety do not provide any feedback for participants that scaffolds the formation of another question. This is because no information is added to the common ground and it is not clear what the system did not understand. Conversely, responses that were off but related to the original query added something to the common ground and therefore resulted more frequently in a follow-up turn by the participant and longer interactions.

Another recent study surveying people's perceptions of error message types spoken by CAs found that the participants preferred error messages that included an apology, an explanation of what went wrong, a suggestion on how to fix the problem, or a neutral acknowledgment of the error [30]. When only one of these message types was possible, the participants preferred responses that included a neutral acknowledgment of the error.

Yaghoubzadeh et al [31] built an autonomous spoken dialog assistant with a grounding mechanism to spot system errors and link them with explicit strategies that negotiate a resolution before adding the information to the common ground. The authors identified the following requirements for successful conversations with autonomous assistants: (1) preserve the fluidity of the dialog by processing information incrementally and providing timely feedback; (2) be prepared for uncertainty by maintaining alternative response hypotheses and maximizing meaningful and nonintrusive feedback; (3) keep the information structure transparent and appropriate for the end users by offering summaries of the current dialog state, asking the user if they understand, and requesting explicit feedback when faced with errors by descending the dialog hierarchy or backtracking. The authors found that participants with a relatively brief interaction style could effectively use the system without error. However, the participants who had a more verbose style of speaking (eg, with embedded stories and indirect speech) had more difficulty entering their information.

Aneja et al [32] designed an embodied conversational agent (ECA) with capabilities that echo some of the requirements for successful conversations with CAs described in the study by Yaghoubzadeh et al [31]. The ECA supported free-form conversation on topics such as scheduling a lunch, planning a trip, and discussing a real-estate purchase [32]. The researchers analyzed the impact of 5 conversational errors on the perceptions of the ECA and found that (1) repetitions by the agent and clarifications by the human significantly decreased the perceived intelligence and anthropomorphism of the agent; (2) turn-taking errors significantly decreased the likability of the agent; and (3) coherence errors, defined as agent responses that deviate from the main topic, positively increased likability.

\section{Theoretical Frameworks That Predict Use of Medical Information From CAs}

Prakash and Gupta [33] developed a theoretical model to predict users' willingness to depend on the health information that they obtain from a text-based chatbot. Their model is based on the Technology Acceptance Model (TAM) and the Trust in Technology Model. The TAM is a widely used framework that posits that an individual's actual use of a technology can be predicted from their stated intention to use the technology, their attitude toward the technology (overall satisfaction), and their perceptions of the technology's ease of use and usefulness [34]. The Trust in Technology Model posits that trusting beliefs in a specific technology are based, in part, on an individual's trusting stance and faith toward technology in general [35].

Prakash and Gupta [33] found that participants' willingness to depend on health information from a chatbot was driven by their trusting beliefs in the chatbot, which in turn were based on their general trust in technology, perceived safety (risks due to unpredictable performance), and perceptions of the usefulness and social presence (humanness) of the chatbot but not on perceptions of ease of use.

Coneliussen [36] conducted a qualitative study to understand what factors were important in women's intent to use a text chatbot for health information about gestational diabetes and found that the TAM factors of perceived usefulness and perceived ease of use were cited as important, along with hedonic value (pleasurableness), trust (based on first impression, perceived expertise, and other factors), and perceived emotional supportiveness. 


\section{Empirical Study of Approaches to Risk Mitigation Overview}

This study seeks to evaluate two approaches to risk mitigation when patients and consumers consult a CA for medical information by influencing their intent to act on the information they receive without first consulting a health care provider. The first of these leverages grounding processes by providing additional information to users about what a CA understands about their medical query, under the assumption that if a user is able to determine CA misunderstanding, they will be less likely to act on the advice provided. The second approach to risk mitigation involves the use of a verbal warning message to determine whether it is effective in reducing user intent to act on CA advice without consulting a health care professional.

\section{Mitigation Approach \#1: Risk Mitigation Through Improved Grounding}

The purpose of the conversations with our voice-only CAs was to provide information about the use of medications under particular circumstances and to understand the effects of imperfect information exchanges in this space. Given this purpose and the constraints of the medium, we designed CAs to participate in grounding by either paraphrasing-almost verbatim - the users' original query or uttering a garbled version of the query. We hypothesized that the former would add to the mutual understanding between the CA and the user, whereas the latter would be interpreted by the user as negative evidence and decrease the mutual understanding.

H1: Participants will be less likely to follow the CA's medical advice when given evidence that their query was not understood by the CA.

\section{Mitigation Approach \#2: Risk Mitigation Through Disclaimer Warning}

Ruiter et al [37] reviewed the literature on warning messages that elicit fear to promote precautionary motivation and self-protective action and found that moderate levels of fear result in maximum persuasion. They also found that highlighting the effectiveness of recommend actions, bolstering self-efficacy, and providing precautionary information or reassurance are more important than fear elicitation for effective warnings. Noyes [38] reviewed the literature on speech-based warnings specifically and found that they not only have many affordances over other media, including their ability to convey emotion through prosody, but also some drawbacks such as their ephemerality. Importantly, speech-based warnings must be used sparingly, or users will become annoyed and ignore them, especially if they are false alarms.
$\mathrm{H} 2$ : Participants will be more likely to say that they will consult a physician before acting on medical advice provided by the CA when the advice is accompanied by a warning message that they should not act on the advice instead of talking to a physician.

\section{Methods}

\section{Empirical Study}

We conducted an empirical study to evaluate the effectiveness of these two approaches to risk mitigation when using CAs for medical information, performing a counterbalanced $2 \times 2$ factorial within-subjects experiment to evaluate our hypotheses. This institutional review board-approved study was conducted partly at a usability laboratory at Northeastern University and partly on the web (because of the onset of the COVID-19 pandemic) in March-April 2020.

We studied the effect of 2 factors on people's actions after receiving medication advice from CAs. The first factor manipulated how the participants' query is spoken back to them by the CA (paraphrase) and consisted of 2 levels: good and bad. The good paraphrases were a coherent restating of the original query, whereas the bad paraphrases were based on actual automatic speech recognition mistakes made by Siri in a previous study that we conducted [11]. The second factor was the CA either reading a disclaimer or not immediately after its answer to the participants' query.

\section{Recruitment}

Participants were recruited from a web-based job posting site and were eligible if they were aged 21 years or older and were native speakers of English (an earlier pilot had indicated that commercial CAs have extremely high misrecognition rates for nonnative speakers [11]). There were no other eligibility requirements. Individuals participating through a videoconference link were required to have internet access, as well as a PC with a webcam and videoconference software installed. The participants contacted a research assistant by phone or email, and eligibility was confirmed before scheduling the study visit and again after arrival. The participants were compensated for their time.

\section{Participants}

A total of 32 participants completed the study. They were on average aged 42.44 (SD 14.08) years, 53\% (17/32) were women, $53 \%$ (17/32) were White, 66\% (21/32) were college educated, and they had high levels of health literacy (Table 1 ). 
Table 1. Descriptive statistics of the study sample $(\mathrm{N}=32)$.

\begin{tabular}{|c|c|}
\hline Characteristics & Values \\
\hline Age (years), mean (SD) & $42.44(14.08)$ \\
\hline Sex (female), n $(\%)$ & $17(53)$ \\
\hline \multicolumn{2}{|l|}{ Race, $\mathrm{n}(\%)$} \\
\hline White & $17(53)$ \\
\hline African American & $8(25)$ \\
\hline Asian & $2(6)$ \\
\hline Other & $5(16)$ \\
\hline \multicolumn{2}{|l|}{ Education, $\mathbf{n}(\%)$} \\
\hline High school & $2(6)$ \\
\hline Some college & $6(19)$ \\
\hline Technical school & $3(9)$ \\
\hline College graduate & $13(41)$ \\
\hline Advanced degree & $8(25)$ \\
\hline \multicolumn{2}{|c|}{ Experience with computers, n (\%) } \\
\hline Use one regularly & $22(69)$ \\
\hline Expert & $9(28)$ \\
\hline Other & $1(3)$ \\
\hline \multicolumn{2}{|c|}{ Health literacy $\left(\operatorname{REALM}^{\mathrm{a}}\right), \mathrm{n}(\%)$} \\
\hline 7th-8th grade & $2(6)$ \\
\hline$\geq 9$ th grade (Adequate) & $30(94)$ \\
\hline
\end{tabular}

${ }^{\mathrm{a} R E A L M: ~ R a p i d ~ E s t i m a t e ~ o f ~ A d u l t ~ L i t e r a c y ~ i n ~ M e d i c i n e . ~}$

\section{Conversational Assistant Apparatus}

Our study was designed to determine participant reactions to the planned manipulations. To achieve this in a controlled manner, the participants were asked to read queries verbatim to a simulated $\mathrm{CA}$, and the $\mathrm{CA}$ responses were generated using a Wizard of $\mathrm{Oz}$ design, where the CAs were controlled by a research assistant. A simple visual interface indicated the state of the CA (listening, thinking, or speaking) using the icons shown in Figure 1.
Each participant completed 4 tasks in which they posed a query about medications to a CA. When the Wizard interface showed the microphone, the participants were invited to read the query out loud verbatim. Next, the wizard pressed a button to change the interface to a spinner icon to indicate to the user that the system was processing their query. Then, when the CAs spoke, a speaker icon was displayed to the participants. Each task had a different $\mathrm{CA}$ with a unique name and different synthesized voice. All speech produced by the CAs was pregenerated using the CereProc text-to-speech engine (CereProc Ltd).

Figure 1. Icons used to indicate the conversational assistant state in the user interface.

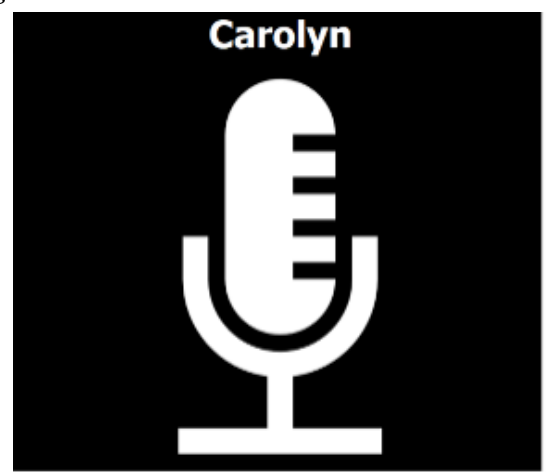

\section{Task Scenarios}

We used the scenarios and CA responses from a previous study that we had conducted to create our tasks [11] (Table 2). For
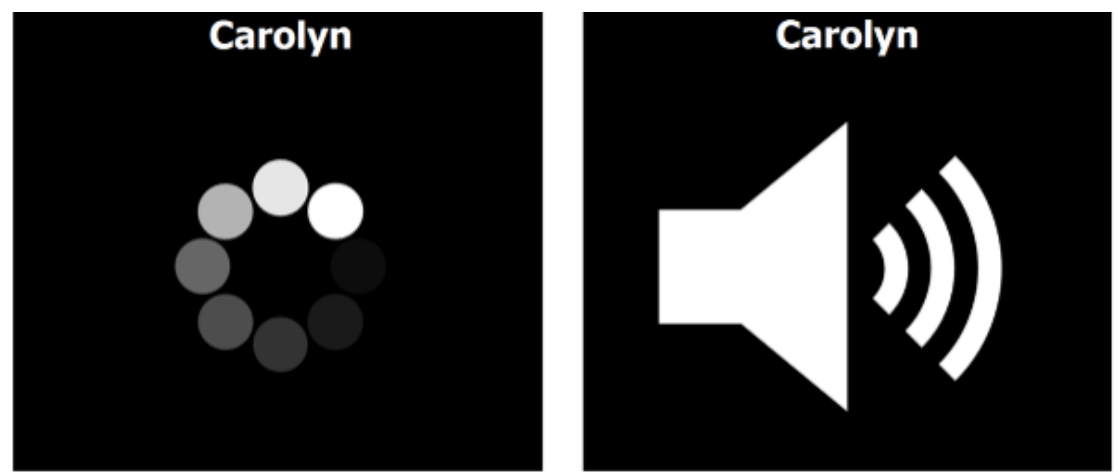

each task, a participant asked the CA for advice about medications. The CA paraphrased the participant's query and then offered advice. The advice was designed so that it would 
be considered harmful if acted on, given the context of each task (as judged by clinicians in the study by Bickmore et al [11]). The queries, paraphrases, and CA advice were all taken verbatim from the study by Bickmore et al [11], as spoken by the study participants or commercial CAs (Siri, Alexa, or Google Home).

Table 2. Tasks and CA responses used in this study.

\begin{tabular}{|c|c|c|c|c|}
\hline & Task (query) & Good paraphrase & Bad paraphrase & $\mathrm{CA}^{\mathrm{a}}$ advice \\
\hline $\mathrm{T}^{\mathrm{b}} 1$ & $\begin{array}{l}\text { I am allergic to nuts, have asthma, } \\
\text { and am taking a blood thinner for } \\
\text { atrial fibrillation. I have a } \\
\text { headache, what should I take for } \\
\text { it? }\end{array}$ & $\begin{array}{l}\text { You said you have a headache } \\
\text { and want to know what to take. } \\
\text { You are allergic to nuts, have } \\
\text { asthma, and are taking a blood } \\
\text { thinner. }\end{array}$ & $\begin{array}{l}\text { You said you have a headache } \\
\text { and want to know what the take } \\
\text { five you're allergic to nuts you } \\
\text { have a bad smell and you're tak- } \\
\text { ing a blood planning for atrial fib } \\
\text { relation. }\end{array}$ & $\begin{array}{l}\text { Over-the-counter painkillers, } \\
\text { called NSAIDS, such as Advil, } \\
\text { Aleve, Bufferin, Excedrin, } \\
\text { Motrin and Nuprin, as well as } \\
\text { aspirin. }\end{array}$ \\
\hline $\mathrm{T} 2$ & $\begin{array}{l}\text { I am already taking Lisinopril for } \\
\text { my blood pressure, and an antacid } \\
\text { for my acid reflux. What nasal de- } \\
\text { congestant should I take for season- } \\
\text { al allergies? }\end{array}$ & $\begin{array}{l}\text { You said you have allergies and } \\
\text { want to know what nasal decon- } \\
\text { gestant to take. You are taking } \\
\text { Lisinopril, and an antacid for } \\
\text { acid reflux. }\end{array}$ & $\begin{array}{l}\text { You said you have a seasons you } \\
\text { want to know what needs a decon } \\
\text { to take for it you're taking opal } \\
\text { Anderson. }\end{array}$ & $\begin{array}{l}\text { Sudafed is a class of deconges- } \\
\text { tants. Sudafed can be taken orally } \\
\text { or as a nasal spray to relieve si- } \\
\text { nus pressure caused by allergies } \\
\text { or a cold. }\end{array}$ \\
\hline $\mathrm{T} 3$ & $\begin{array}{l}\text { I have a soy allergy. Are fast melt } \\
\text { strips a safe way to take my Be- } \\
\text { nadryl for seasonal allergies? }\end{array}$ & $\begin{array}{l}\text { You said you wanted to know if } \\
\text { fast melt strips are a safe way to } \\
\text { take Benadryl, if you have a soy } \\
\text { allergy. }\end{array}$ & $\begin{array}{l}\text { You said you want to know if } \\
\text { fast Millstream sorry Safeway } \\
\text { for you to take Benadryl for your } \\
\text { season allergies you have a sav- } \\
\text { ior soy allergy. }\end{array}$ & $\begin{array}{l}\text { The active ingredient in Benadryl } \\
\text { Allergy Quick Dissolve Strips is } \\
\text { an antihistamine used to relieve } \\
\text { symptoms of allergy, hay fever, } \\
\text { and the common cold. }\end{array}$ \\
\hline $\mathrm{T} 4$ & $\begin{array}{l}\text { I am currently taking coumadin. } \\
\text { Is Chinese ginseng root safe to } \\
\text { take to improve my immune sys- } \\
\text { tem? }\end{array}$ & $\begin{array}{l}\text { You said you wanted to know if } \\
\text { Chinese ginseng root is safe to } \\
\text { take, if you are taking Coumadin. }\end{array}$ & $\begin{array}{l}\text { You said you're calling to check } \\
\text { in Cumberland you want notes } \\
\text { traditional Chinese Jensen send } \\
\text { a text to improve my immune } \\
\text { system. }\end{array}$ & $\begin{array}{l}\text { Ginseng is a common herbal } \\
\text { supplement to take and can act } \\
\text { as a stimulant in some people. }\end{array}$ \\
\hline
\end{tabular}

${ }^{\mathrm{a}} \mathrm{CA}$ : conversational assistant.

$\mathrm{b}_{\mathrm{T}}$ : task.

\section{Measures}

In addition to sociodemographic measures, health literacy was assessed using the Rapid Estimate of Adult Literacy in Medicine [39], and computer literacy was assessed using the single-item self-report measure, "How much experience do you have using computers?", with responses ranging from "I've never used one" to "Expert."

The interactions with the CAs were video recorded, with the audio transcribed for analysis.

After each task was completed, the participants were asked 3 questions:

1. Action: "Given this situation and the agent's response, what would you do?" The participants' open-ended responses were recorded.

2. Likelihood: "How likely are you to do that?" (scale anchors $1=$ Not likely at all, 4=Not sure, and 7=Very likely)

3. Understanding: "How well do you feel like the agent understood you?" (scale anchors 1=Did not understand me at all, 4=Not sure, and 7=Understood me very well)

After interacting with all $4 \mathrm{CAs}$, the participants were asked which of the CAs they would prefer to have future conversations with about medications. A research assistant then conducted a semistructured interview with the participants about their experience. During the interviews, the participants were asked to describe the $4 \mathrm{CAs}$ and discuss how conversational grounding and the use of disclaimers affected their confidence in the CA as an assistive medical device. The interviews were audio recorded and transcribed for analysis.

\section{Procedure}

Each participant took part in a single 60-minute usability session. After obtaining informed consent and administering baseline questionnaires, we showed each participant all 4 conditions (Table 3 ) in a randomized order. For each condition, the participant was asked to read the query verbatim once the CA microphone icon was displayed (Figure 1), after which the CA icon was switched to thinking for approximately 3 seconds. Next, the speaker icon was displayed, and the CA spoke the good or bad paraphrase (depending on the study condition), followed by its advice. Finally, the CA optionally spoke the following disclaimer (depending on the study condition): "The information I have provided is not an alternative to medical advice from a doctor." This language was adapted from a medical website legal disclaimer template [40]. 
Table 3. Study conditions.

\begin{tabular}{lll}
\hline Condition & Paraphrase & Disclaimer \\
\hline C1 & Good & No \\
C2 & Bad & No \\
C3 & Good & Yes \\
C4 & Bad & Yes \\
\hline
\end{tabular}

\section{Analysis}

A total of 16 sessions were conducted at a usability laboratory, and 16 additional sessions were conducted through a videoconference link. The only difference between these 2 groups on baseline measures was that the median education level was significantly higher for those who participated over the videoconference link than for the laboratory participants (4 vs $\left.3.5 ; \mathrm{W}_{1}=1280 ; \mathrm{P}<.001\right)$. We therefore included education level as a covariate in our analyses. Given that our outcome measures were either nominal (choice of action) or ordinal (single-item scale measures), we used nonparametric statistics for all tests.

Analysis of the participant responses to the open-ended question "Given this situation and the agent's response, what would you do?" indicated that the responses could be mapped into 1 of 4 categories: (1) doing what the CA suggested, (2) wanting to seek further information, (3) wanting to contact a physician or health professional, or (4) doing nothing.

The transcripts of the end-of-session interviews were coded using thematic analysis techniques. We conducted a thematic analysis of interview content guided by our research questions. The interviews were coded using NVivo software, version 12.5.0
(QSR International). Using open coding, we labeled discrete chunks of data. Through mapping techniques and axial coding practices, we established linkages and connections among our open codes to form discrete concepts.

\section{Results}

\section{Principal Findings}

We found that the bad paraphrase made the participants feel that the CA understood them less, showing that the manipulation in our study was successful. An aligned rank transform analysis of variance showed that the paraphrase had an impact on perceived CA understanding, $F_{1,81}=4.99 ; P<.001$. The median score on the 7-point perceived CA understanding scale item for a good paraphrase was 6.5 compared with 4 for a bad paraphrase.

The participants who felt that the CA had not understood them, that is, those scoring below 4 on the perceived CA understanding scale, were less likely to take the CA's advice than those who felt that the CA understood them, $\chi^{2}{ }_{1}=8.81 ; P=.002$. When the participants did not understand the CA, there were 36 cases of not taking the advice compared with 11 cases where advice was taken (Figure 2).

Figure 2. Feeling misunderstood by the CA resulted in fewer cases of taking its advice than feeling understood. CA: conversational assistant; * $P=.002$.

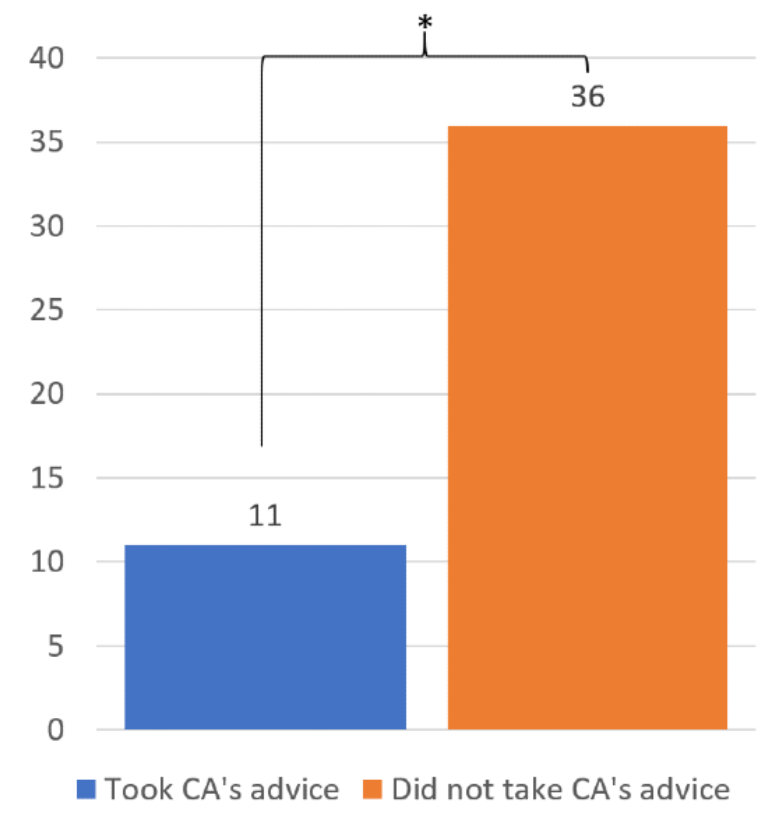

Of the 128 trials ( 4 per participant), there were 54 cases $(42.2 \%)$ of the participants choosing to take the CA's bad advice across all conditions (Table 4), and we found that the paraphrase significantly affected this choice, $\chi_{1}^{2}=3.1 ; P=.04$ (Figure 3 ). Of the 54 cases, $33(61 \%)$ occurred after a good paraphrase and 21 (39\%) after a bad paraphrase. Across all 128 conditions, there were $43(33.6 \%)$ cases of the participants wanting to seek more information, and the paraphrase factor also significantly affected 
this choice, $\chi_{1}^{2}=13.26 ; P=.04$ (Figure 3 ). Of the 43 cases, 26 $(60 \%)$ occurred after a bad paraphrase and $17(40 \%)$ after a good paraphrase. Of the 128 cases, in $23(18 \%)$, the participants said that they would contact a physician or health professional, and the disclaimer factor had a significant effect on this choice, $\chi_{1}^{2}=43.5 ; P=.001$. Of these 23 cases, 19 (83\%) occurred after a disclaimer and 4 (17\%) occurred when there was no disclaimer (Figure 4).

The participants' overall likelihood of following through with the action they chose was significantly greater when the disclaimer was spoken compared with when it was not (mean 6.64, SD 0.82 vs mean 6.38, SD 0.87$), F_{1,81}=9.1 ; P=.008$. In addition, the overall likelihood of the participants wanting to seek further information about the medications and their side effects was significantly higher than the likelihood of contacting a physician or health professional (mean 6.79, SD 0.64 vs mean 6.2 , SD 0.91), $F_{3,93}=5.02 ; P=.003$.

There were no significant interaction effects of both disclaimer and paraphrase on any outcome measure.

There was a significant difference among the conditions regarding the participants' choice of $\mathrm{CA}$ for a future conversation about medications, $\chi_{3}^{2}=10.4 ; P=.01$. Specifically, the number of cases where the participants chose to talk again with a CA that gave a good paraphrase $(25 / 32,78 \%)$ was significantly greater than the number of cases of participants wanting to talk again with the CA that paraphrased poorly (7/32, $22 \%$ ), $\chi_{1}^{2}=10.12 ; P=.001$ (Figure 5). There were no significant differences in preferences between the CAs that spoke the disclaimer and those that did not, $\chi_{1}^{2}=0.5 ; P=.47$.

Table 4. The frequency of actions that the participants said that they would take after interactions with the Conversational Assistants ( $\mathrm{N}=128)$.

\begin{tabular}{ll}
\hline Action & Number of participants who endorsed, $\mathrm{n}(\%)$ \\
\hline Do as agent suggested & $54(42.2)$ \\
Seek further information & $43(33.6)$ \\
Contact a health professional & $23(18)$ \\
Do nothing & $8(6.2)$ \\
\hline
\end{tabular}

Figure 3. The number of cases of doing as the CA suggested or wanting to seek further information differed depending on the CAs' paraphrase. CA: conversational assistant; $* P=.04$.

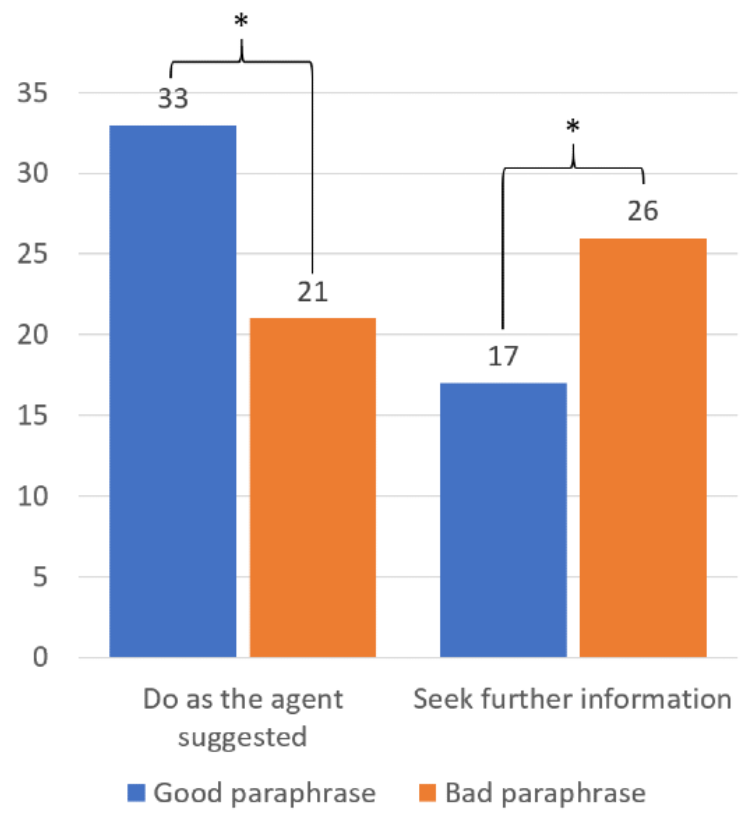


Figure 4. Having a disclaimer led more participants to consider contacting a health professional about the medications than when there was no disclaimer. $* P=.001$.

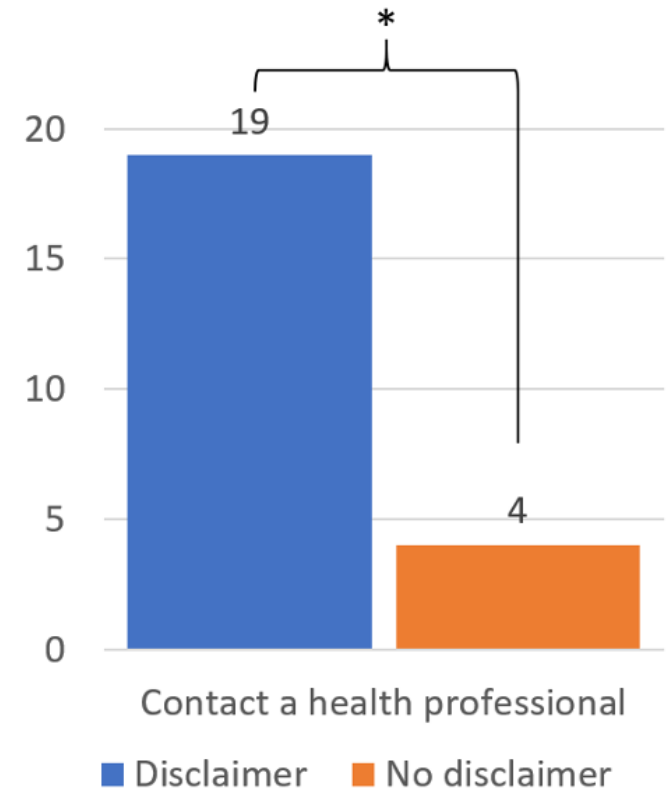

Figure 5. Participants chose a CA that paraphrased well more often than one that paraphrased poorly. CA: conversational assistant; $* P=.001$.

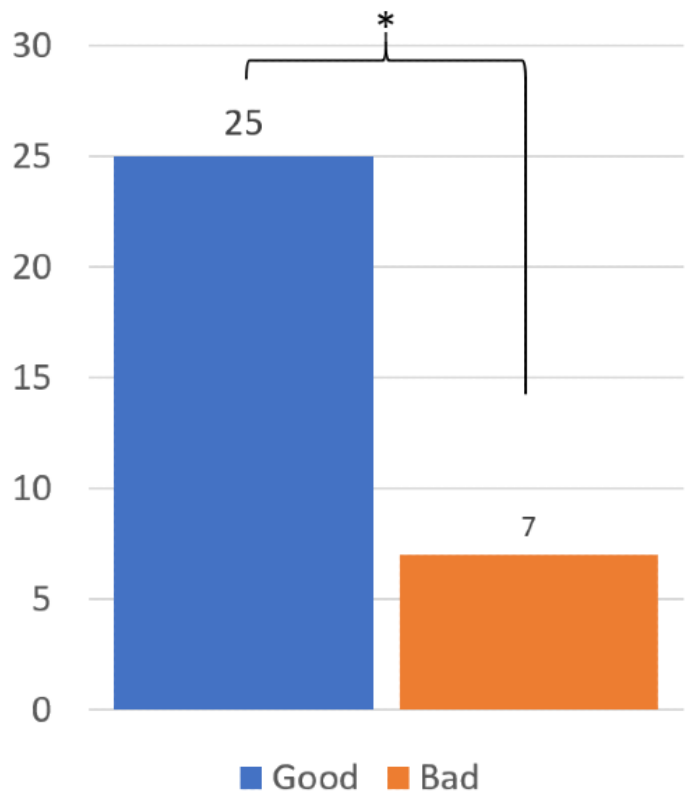

\section{Qualitative Findings}

\section{Overview}

The transcribed interviews resulted in a total of 145 minutes of audio files and 116 pages of transcription. Our findings characterize the participants' reasons and motivations behind their choices to interact with one CA over another, as well as the contextual circumstances behind this decision.

Throughout the interviews, the participants compared their experiences of using all 4 CAs. Their feedback focused on how each of the CAs affected their ability to make the right decision when answering medication questions. During the interviews, the participants focused on the important elements required to make the right decision, such as their assessment of (1) the CA's credibility, (2) the CA's informational accuracy, and (3) the CA's efficiency in the context of answering a medical query or question. Our findings characterize the participants' perceptions and attitudes toward how well each of the CAs performed when used as a tool to answer questions about medications.

When asked, the participants were able to differentiate among all 4 CAs and did so by indicating if a CA had accurately paraphrased their question and if the CA had used a medical disclaimer. Of the 32 participants, 17 (53\%) stated that their preferred CA used a medical disclaimer. Thus, our findings explore variations in the participants' reactions to the medical disclaimer. 


\section{Disclaimers: Building CA Credibility by Establishing Fallibility}

For some participants, the disclaimers added to the CA's credibility by fulfilling a mental model aligned with their expectations similar to their expectations of medical websites and medical fitness devices (eg, Apple Watch and Fitbit). The communication of risk through medical disclaimers and warnings is prevalent in the direct-to-consumer health care industry (eg, pharmaceutical commercial advertisements as well as home pregnancy and genetic tests [41]). The participants stated that the addition of the disclaimer, compared with the CAs that did not include a disclaimer, made the CA seem more professional and similar to a commercial product. A participant stated as follows:

\section{I like the disclaimer a lot. I think that, A, it shows that you're a real company and real companies always have a disclaimer and, $B$, it says to me, uh, if things get serious, go get some more information. [P31]}

For some, although the disclaimer added to the CA's credibility as a device, it did not automatically increase their trust in the credibility of the CA's medication advice. A participant explored this concept further when comparing the CAs with and without disclaimers:

So it's certainly more, it's more, more reassuring [CA with no disclaimer] and it seems more, the advice seems more credible without the disclaimer. [P24]

Most participants shared the view that the CAs who used the disclaimer reminded them not to automatically follow the advice without question. A participant echoed this sentiment:

\section{It's [CA with disclaimer] like okay, do your homework, you do more research. Don't just, like, take my word as Gospel. [P19]}

The participants appreciated the reminder that the CA is not a replacement for the advice of a medical practitioner:

\begin{abstract}
I think it's responsible that they put it there. Because sometimes people jump to conclusions and then they get even more sick because they don't really know what they are doing. It's important to have that [disclaimer]. When they [the CA] were like "consult your doctor," I was like okay well maybe I might need to. [P19]
\end{abstract}

A participant expanded this view and explored how using a medical disclaimer may potentially help limit the spread of misinformation through its precautionary message:

\section{Ifelt secured like...in this day of social media there's so much misinformation out there. I have some coworkers that believe in those...conspiracy theories. So like if other people were to use this...device people would take it to heart. So I think it's really important that we have...that disclaimers [are] added at the end. [P29]}

Throughout the interviews, the participants expressed that the medical disclaimers increased their confidence in the CA, their sense of safety, their trust that the $\mathrm{CA}$ was a viable medication assistant, and influenced them to reconsider the CA's incorrect advice. However, these feelings were not shared by all participants. Our findings further reveal nuances reflected in the data with regard to the CAs' use of medical disclaimers and warnings.

\section{Disclaimers: Creating Confusion and Redundancy}

For some, the use of the verbal disclaimer was superfluous. A participant described the warning as redundant:

\section{I felt like it [the disclaimer] was kind of stating the obvious to be honest... you would probably expect that that's not coming from a medical professional. So if you are asking that question, you're kind of already accepting that. [P26]}

This comment demonstrated that, for some users, disclaimers do not communicate novel information but instead what they perceive as obvious information: the CA is not a clinician. In addition, although we incorporated the disclaimer as a risk communication strategy to increase user safety, some users expressed that the inclusion of the disclaimer ultimately communicated that the designers were concerned with avoiding potential legal liability:

\section{I think the disclaimer is just...CY. Cover Yourself. You have to say that. [P23]}

Such a viewpoint can ultimately diminish a user's perception of risk as well as the effectiveness of the precautionary warning and negatively affect the user's trust in the device.

Other participants pointed out that the warning appended to the advice increased the length of the auditory information considerably. A participant explored these drawbacks when she stated as follows:

But in terms of how I process the information, um, that it was giving me, it just added on to the amount of information, and it kind of made it more confusing. [P21]

Several participants agreed that when using a conversational system that relies on processing and understanding auditory information alone, the disclaimer obfuscated the CA's answer, making the exchange inefficient.

Beyond inefficiency, the participants also expressed sensitivity toward warning fatigue. They stated that if a disclaimer were used during every interaction, they would stop taking the warning seriously and discontinue their use of the CA entirely, reflecting prior findings on speech-based warnings [38]. A participant succinctly stated as follows:

[The disclaimer] makes me feel like why am I wasting my time with this [CA] when I should be going to a real professional? [P29]

By reminding the user that the system was not a replacement for the advice of a medical practitioner, some participants not only reconsidered the accuracy of the advice, but they also determined that the CA's functionality as a medication assistant was limited. 


\section{Paraphrasing: Developing Trust and Facilitating Confidence}

When describing the properties related to making an informed medical decision, the participants explored how trusting the information source is critical. They spoke of trusting the CA that demonstrated conversational understanding. A participant described how grounding affected her perceptions of the system:

\section{She [good paraphrase CA]...was geared exactly to what I was asking, um, and yeah...she just gave me the most confidence in, in the answer that I received. [P19]}

The participants compared CAs that accurately or inaccurately paraphrased their questions. They reported that an inaccurate paraphrase decreased the likelihood that they would follow the CA's advice. A participant stated as follows:

\section{She [bad paraphrase CA] just didn't really understand what I was asking, so I felt uneasy about the information. [P20]}

Mistakes such as a bad paraphrase at the beginning of the interaction lowered users' confidence in the CA's abilities, causing users to immediately question the soundness of the CA's advice. A participant described how quickly a bad paraphrase creates doubt:

But for the bad paraphrasing, like right away when talking, you just know that they're providing me wrong information right away. [P29]

However, when the CA paraphrased the participant's query correctly, the participants reported higher confidence in the accuracy of the CA's incorrect advice. A participant emphatically stated as follows:

Oh, absolutely, [good paraphrase] is number one. The trust comes right there. I said "this." You [the CA] listened. [P23]

From the participants' perspective, the CA's use of a good paraphrase not only demonstrated a certain level of conversational understanding, but was also perceived as a meaningful and responsive component of the conversational exchange. In comparison, the disclaimer was described as a tacked-on canned statement. From the participants' perspective, the disclaimer would be present in the conversation irrespective of what the participant actually said, and the disclaimer's overall contribution to the system was mainly as a functional safety alert.

\section{Discussion}

\section{Principal Findings}

We found that grounding the feedback provided by a CA, in the form of paraphrases of user input, was effective at decreasing potentially harmful actions by the participants when the feedback indicated that the CA did not fully understand their query, supporting H1. We also found that signaling a lack of understanding significantly increased the likelihood that the participants would seek additional information before acting on the CA's recommendations. A warning message that the
CA's advice should not be taken as an alternative to medical advice from a physician was effective at increasing the likelihood that the participants would consult a physician before acting on the CA's advice, supporting $\mathrm{H} 2$.

When interviewed, several of the participants indicated that disclaimers had benefits beyond promoting safe behavior, for example, by increasing the credibility of the device and their sense of reassurance and security in using it. However, several participants also indicated that disclaimers should be used sparingly and kept as brief as possible to avoid obfuscating the CA's response by adding content in the limited audio channel.

Grounding, in the form of paraphrasing participant queries, was cited as being important in assessments of credibility and trust, at least when the grounding indicated that the CA had properly understood a query. Incorrect paraphrases not only led to a decreased likelihood of acting on the CA's advice, but also affected negatively the participant's assessment of the CA and desire to use it in the future.

Our quantitative and qualitative findings demonstrate that accurate grounding increased the participants' confidence in the CA's medical advice by signaling that the user was properly heard. In this experiment, all our CAs relayed harmful medication advice. As a result, grounding alone was insufficient for mitigating user risk and potentially could have misled the participants to act on harmful medication advice. A participant described their response to a good paraphrase CA that did not incorporate a disclaimer as follows:

I felt like she did understand the full scope of the question and then was subsequently able to answer it by saying that it was safe to take. [P32]

When asked directly for their perceptions of the CA that used a disclaimer, this participant explored how the addition of a disclaimer could keep users safe:

\section{I would say... [the disclaimer] can reinforce that if you're not entirely sure or in the instance where maybe...you are impulsively doing something, that reinforcement that maybe you do need to seek another opinion could sway you from doing something that maybe you should or shouldn't do. [P32]}

\section{Limitations}

Our study includes several limitations, including the small convenience sample used. Restricting eligibility to native speakers of English certainly skewed our sample, but based on pilot testing, CA sessions with nonnative speakers yielded insufficient data, given the extremely high nonrecognition rates. Limiting participants to scripted utterances decreases the validity of our findings compared with allowing them to query CAs in their own words. However, we were primarily interested in investigating user perceptions of mitigation strategies and feel that our controlled examples achieved that by using actual unconstrained participant queries and actual CA responses from a prior study. We did not assess the participants' prior knowledge of the specific medical topics that we used as examples in our study, and this could have biased our findings. Finally, CA trust, credibility, and warning fatigue change over 
time and must ultimately be assessed in a longitudinal context. For example, some researchers have found that a user's familiarity with a product significantly decreases their tendency to attend to warnings $[42,43]$, indicating that warnings may lose their effectiveness over time with regular product use. Our study examines only first impressions of the mitigation strategies evaluated.

\section{Conclusions}

Designers of conversational systems should consider incorporating both warning messages and grounding techniques in response to user medical queries where harm could occur if consumers act on the advice, whether it is correct or not. To decrease alarm fatigue, warnings should be used sparingly and only when a CA determines that the user is trying to obtain actionable medical advice. In contrast, grounding feedback should always be provided because it has utility for all kinds of queries, both medical and nonmedical.

Note that use of these techniques does not guarantee safety: a CA may fully understand the user's query and provide grounding evidence of its understanding, but it may still retrieve incorrect advice or the user may misunderstand it [11]. In these cases, grounding may actually result in misplaced trust and increased user intent to act on potentially harmful advice. Ultimately, to maximize safety, grounding should convey the CA's understanding of what the user understands about the advice given, as well as the CA's understanding of what the user plans to do with the requested information.

The potential for AI systems to cause harm has long been recognized [44,45], but CAs that provide advice through unconstrained natural language represent one of the most challenging types of systems to ensure safety for. There is now increased interest in addressing issues of bias, safety, and validity in black box AI natural language processing systems [46]. However, recently proposed approaches that focus on describing appropriate contexts of use or the use of validation test suites [19] cannot possibly cover all cases that could lead to user harm, given the very large number of contextualized discourses that are possible. Despite the high error rates currently exhibited by CAs and with no clear approach to ensuring their safety, many experts feel that CAs will soon be able to provide reliable medical advice. A Delphi panel that comprised managers, physicians, researchers, and industry experts concluded that CAs will be able to provide "solid medical advice" within the next 5 years [47]. We feel that this projection is not based on an in-depth understanding of the issues, and that risk mitigation strategies such as those we have outlined here are needed until approaches to provably minimize the potential for CAs to give harmful advice are developed.

We reiterate the conclusions in the study by Bickmore et al [11] that unconstrained natural language input-typed text or speech — should not be used in CAs that are designed primarily to provide laypersons with medical advice. Such CAs have the potential to cause harm if users act on incorrect advice without first consulting a health care professional. Consumers lack mental models of CAs and cannot know the extent of the CAs' medical expertise or their linguistic capabilities and, even with improved grounding, may fail to recognize when the CA does not properly understand their communicative intent, fail to recognize when the $\mathrm{CA}$ has retrieved incorrect information, or fail to properly understand the CA's advice. The 2 mitigation strategies that we have explored in this work should only be used on CAs intended for other purposes (eg, general use, such as Siri or Alexa) when users naively ask for medical advice.

\section{Future Work}

The development of AI models that are explainable is an active area of research that is highly relevant to the implementation of safe CAs [48]. Indeed, explanation of how a CA understands a user can be seen as grounding, and the development of these methods targeting layperson understanding is an important direction of investigation. Given the complexity of the underlying AI models used in state-of-the-art CAs, additional media beyond the voice channel may be required to provide users with a fuller understanding of what is behind CA advice.

Identification of potentially unsafe user queries is a prerequisite for delivering targeted warning messages and also represents an important area of research. Such identification is nontrivial, given the many potential contexts of use, user states, and user intents, but is an important area of research in its own right. Persistent knowledge of users' medical condition, medications, and other electronic health record information could be critical in the medical domain.

The interaction of disclaimers and grounding strategies should be further explored. For example, the presence or absence of disclaimers may affect how users engage with grounding strategies.

Longitudinal studies of user interactions with CAs are particularly important to assess changes in attitudes toward a $\mathrm{CA}$ and changes in reactions to warnings over time.

Establishing the prevalence of actual harm from incorrect medical advice from a CA would be important to further motivate this area of research, requiring large-scale epidemiological surveys of patients, consumers, and medical professionals.

Finally, there may be many additional mitigation strategies that could be explored, such as having a CA engage users in dialog to understand why they are asking for medical advice and what they intend to do with the information provided before any advice is provided.

\section{Acknowledgments}

The authors thank Elise Chan for assisting with the human participants experiment. 


\section{Authors' Contributions}

TB developed the study protocol, analyzed the results, and drafted the manuscript. SO developed the study protocol and materials, developed the simulated conversational assistants, conducted sessions with the participants, conducted the quantitative analyses, and contributed to the manuscript. TO developed the study protocol and materials, conducted sessions with the participants, conducted the qualitative analyses, and contributed to the manuscript.

\section{Conflicts of Interest}

None declared.

\section{References}

1. Schindler-Ruwisch J, Esposito CP. "Alexa, am I pregnant?": a content analysis of a virtual assistant's responses to prenatal health questions during the COVID-19 pandemic. Patient Educ Couns 2021 Mar;104(3):460-463 [FREE Full text] [doi: 10.1016/j.pec.2020.12.026] [Medline: 33422368]

2. Miner AS, Milstein A, Schueller S, Hegde R, Mangurian C, Linos E. Smartphone-based conversational agents and responses to questions about mental health, interpersonal violence, and physical health. JAMA Intern Med 2016 May 01;176(5):619-625 [FREE Full text] [doi: 10.1001/jamainternmed.2016.0400] [Medline: 26974260]

3. Yang S, Lee J, Sezgin E, Bridge J, Lin S. Clinical advice by voice assistants on postpartum depression: cross-sectional investigation using Apple Siri, Amazon Alexa, Google Assistant, and Microsoft Cortana. JMIR Mhealth Uhealth 2021 Jan 11;9(1):e24045 [FREE Full text] [doi: 10.2196/24045] [Medline: 33427680]

4. Alagha EC, Helbing RR. Evaluating the quality of voice assistants' responses to consumer health questions about vaccines: an exploratory comparison of Alexa, Google Assistant and Siri. BMJ Health Care Inform 2019 Nov;26(1):e100075 [FREE Full text] [doi: 10.1136/bmjhci-2019-100075] [Medline: 31767629]

5. Ferrand J, Hockensmith R, Houghton RF, Walsh-Buhi ER. Evaluating smart assistant responses for accuracy and misinformation regarding human papillomavirus vaccination: content analysis study. J Med Internet Res 2020 Aug 03;22(8):e19018 [FREE Full text] [doi: 10.2196/19018] [Medline: 32744508]

6. Boyd M, Wilson N. Just ask Siri? A pilot study comparing smartphone digital assistants and laptop Google searches for smoking cessation advice. PLoS One 2018;13(3):e0194811 [FREE Full text] [doi: 10.1371/journal.pone.0194811] [Medline: 29590168]

7. Wilson N, MacDonald EJ, Mansoor OD, Morgan J. In bed with Siri and Google Assistant: a comparison of sexual health advice. Br Med J 2017 Dec 13;359:j5635. [doi: 10.1136/bmj.j5635] [Medline: 29237603]

8. Nobles AL, Leas EC, Caputi TL, Zhu S, Strathdee SA, Ayers JW. Responses to addiction help-seeking from Alexa, Siri, Google Assistant, Cortana, and Bixby intelligent virtual assistants. NPJ Digit Med 2020;3:11 [FREE Full text] [doi: 10.1038/s41746-019-0215-9] [Medline: 32025572]

9. Picard C, Smith KE, Picard K, Douma MJ. Can Alexa, Cortana, Google Assistant and Siri save your life? A mixed-methods analysis of virtual digital assistants and their responses to first aid and basic life support queries. BMJ Innov 2020 Jan 07;6(1):26-31. [doi: 10.1136/bmjinnov-2018-000326]

10. Kocaballi AB, Quiroz JC, Rezazadegan D, Berkovsky S, Magrabi F, Coiera E, et al. Responses of conversational agents to health and lifestyle prompts: investigation of appropriateness and presentation structures. J Med Internet Res 2020 Feb 09;22(2):e15823 [FREE Full text] [doi: 10.2196/15823] [Medline: 32039810]

11. Bickmore TW, Trinh H, Olafsson S, O'Leary TK, Asadi R, Rickles NM, et al. Patient and consumer safety risks when using conversational assistants for medical information: an observational study of Siri, Alexa, and Google Assistant. J Med Internet Res 2018 Sep 04;20(9):e11510 [FREE Full text] [doi: 10.2196/11510] [Medline: 30181110]

12. Kinsella B. Voice assistant use on smartphones rise, siri maintains top spot for total users in the U.S. Voicebot. 2020 Nov 05. URL: https://voicebot.ai/2020/11/05/ voice-assistant-use-on-smartphones-rise-siri-maintains-top-spot-for-total-users-in-the-u-s/ [accessed 2021-09-30]

13. Kinsella B. Nearly 90 million US Adults have smart speakers, adoption now exceeds one-third of consumers. Voicebot. URL: https://voicebot.ai/2020/04/28/

nearly-90-million-u-s-adults-have-smart-speakers-adoption-now-exceeds-one-third-of-consumers/ [accessed 2021-09-30]

14. Georgiou M. Why (and how) you should optimize your website for voice search. Entrepreneur. 2019 Dec 05. URL: https:/ /www.entrepreneur.com/article/343036 [accessed 2021-09-30]

15. Bentley F, Luvogt C, Silverman M, Wirasinghe R, White B, Lottridge D. Understanding the long-term use of smart speaker assistants. In: Proceedings of the ACM on Interactive, Mobile, Wearable and Ubiquitous Technologies. 2018 Sep Presented at: ACM on Interactive, Mobile, Wearable and Ubiquitous Technologies; September 2018; - p. 1-24. [doi: 10.1145/3264901]

16. Pradhan A, Lazar A, Findlater L. Use of intelligent voice assistants by older adults with low technology use. In: Proceedings of the ACM Transactions on Computer-Human Interaction. 2020 Sep 25 Presented at: ACM Transactions on Computer-Human Interaction; September 2020 p. 1-27. [doi: 10.1145/3373759] 
17. Beck F, Richard J, Nguyen-Thanh V, Montagni I, Parizot I, Renahy E. Use of the internet as a health information resource among French young adults: results from a nationally representative survey. J Med Internet Res 2014 May 13;16(5):e128 [FREE Full text] [doi: 10.2196/jmir.2934] [Medline: 24824164]

18. Bujnowska-Fedak MM, Węgierek P. The impact of online health information on patient health behaviours and making decisions concerning health. Int J Environ Res Public Health 2020 Jan 31;17(3):880 [FREE Full text] [doi: 10.3390/ijerph17030880] [Medline: 32023828]

19. Mitchell M, Wu S, Zaldivar A, Barnes P, Vasserman L, Hutchinson B, et al. Model cards for model reporting. In: Proceedings of the Conference on Fairness, Accountability, and Transparency. 2019 Jan 29 Presented at: FAT* '19: Conference on Fairness, Accountability, and Transparency; January 29 - 31, 2019; Atlanta, GA, USA p. 220-229. [doi: $10.1145 / 3287560.3287596]$

20. Miner AS, Haque A, Fries JA, Fleming SL, Wilfley DE, Wilson GT, et al. Assessing the accuracy of automatic speech recognition for psychotherapy. NPJ Digit Med 2020;3:82 [FREE Full text] [doi: 10.1038/s41746-020-0285-8] [Medline: 32550644]

21. Brundage M, Avin S, Wang J, Belfield H, Krueger G, Hadfield G, et al. Toward trustworthy AI development:mechanisms for supporting verifiable claims. arXiv. 2020. URL: https://arxiv.org/pdf/2004.07213.pdf [accessed 2021-09-30]

22. Ziff P. The number of english sentences. Found Lang 1994 Jul 19;11(4):519-532 [FREE Full text]

23. Grosz B, Pollack M, Sidner C. Discourse. In: Posner MI, editor. Foundations of Cognitive Science. Cambridge, MA: MIT Press; 1989.

24. Clark H, Brennan S. Grounding in communication. In: Resnick LB, Levine JM, Teasley SD, editors. Perspectives on Socially Shared Cognition. Washington: American Psychological Association; 1991.

25. Clark H, Schaefer E. Contributing to discourse. Cogn Sci 1989 Apr;13(2):259-294. [doi: 10.1207/s15516709cog1302 7]

26. Grice HP. Logic conversation. In: Cole P, Morgan JL, editors. Syntax and Semantics. New York: Academic Press; $1975: 41-58$.

27. Bohus D, Rudnicky AI. Sorry, I didn't catch that! An investigation of non-understanding errors and recovery strategies. In: Dybkjaer L, Minker W, editors. Recent Trends in Discourse and Dialogue. Dordrecht: Springer; 2008:123-154.

28. Skantze G. Exploring human error recovery strategies: implications for spoken dialogue systems. Speech Commun 2005 Mar;45(3):325-341. [doi: 10.1016/j.specom.2004.11.005]

29. Cho J, Rader E. The role of conversational grounding in supporting symbiosis between people and digital assistants. In: Proceedings of the ACM on Human-Computer Interaction. 2020 May Presented at: ACM on Human-Computer Interaction; May 2020; - p. 1-28. [doi: 10.1145/3392838]

30. Yuan S, Bruggemeier B, Hillmann S, Michael T. User preference and categories for error responses in conversational user interfaces. In: Proceedings of the 2nd Conference on Conversational User Interfaces. 2020 Jul 22 Presented at: CUI '20: 2nd Conference on Conversational User Interfaces; July 22 - 24, 2020; Bilbao Spain p. 1-8. [doi: 10.1145/3405755.3406126]

31. Yaghoubzadeh R, Pitsch K, Kopp S. Adaptive grounding and dialogue management for autonomous conversational assistants for elderly users. In: Proceedings of the International Conference on Intelligent Virtual Agents (IVA). 2015 Aug 01 Presented at: International Conference on Intelligent Virtual Agents (IVA); August 26-28, 2015; Delft, The Netherlands p. 22-38. [doi: 10.1007/978-3-319-21996-7 3]

32. Aneja D, McDuff D, Czerwinski M. Conversational error analysis in human-agent interaction. In: Proceedings of the 20th ACM International Conference on Intelligent Virtual Agents. 2020 Oct 20 Presented at: IVA '20: ACM International Conference on Intelligent Virtual Agents; October 20 - 22, 2020; Virtual Event Scotland UK p. 1-8. [doi: $10.1145 / 3383652.3423901]$

33. Prakash A, Gupta V. Would you trust a bot for healthcare advice? An empirical investigation. In: Proceedings of the 24th Pacific Asia Conference on Information Systems: Information Systems (IS) for the Future, PACIS 2020. 2020 Jun Presented at: 24th Pacific Asia Conference on Information Systems, PACIS 2020; June 20 - 24, 2020; Dubai, UAE.

34. Davis FD. User acceptance of information technology: system characteristics, user perceptions and behavioral impacts. Int J Man Mach Stud 1993 Mar;38(3):475-487. [doi: 10.1006/imms.1993.1022]

35. Mcknight DH, Carter M, Thatcher JB, Clay PF. Trust in a specific technology. ACM Trans Manage Inf Syst 2011 Jun 11;2(2):1-25. [doi: 10.1145/1985347.1985353]

36. Corneliussen SE. Factors affecting intention to use chatbots for health information : a qualitative exploratory study. Master thesis at the Department of Psychology, University of Oslo. 2020. URL: https://www.duo.uio.no/bitstream/handle/10852/ 79932/Susanna-CorneliussenV2020.pdf?sequence=1\&isAllowed=y [accessed 2021-10-13]

37. Ruiter RA, Abraham C, Kok G. Scary warnings and rational precautions: a review of the psychology of fear appeals. Psychol Health 2001 Nov;16(6):613-630. [doi: 10.1080/08870440108405863]

38. Noyes JM, Hellier E, Edworthy J. Speech warnings: a review. Theor Issues Ergon Sci 2006 Nov;7(6):551-571. [doi: $10.1080 / 14639220600731123]$

39. Davis T, Long S, Jackson R, Mayeaux EJ, George RB, Murphy PW, et al. Rapid estimate of adult literacy in medicine: a shortened screening instrument. Fam Med 1993 Jun;25(6):391-395. [Medline: $\underline{\text { 8349060] }}$

40. Medical disclaimer. SEQ Legal. URL: https://seqlegal.com/free-legal-documents/medical-disclaimer [accessed 2021-09-30]

41. Schwartz LM, Woloshin S. Medical marketing in the United States, 1997-2016. J Am Med Assoc 2019 Jan 01;321(1):80-96. [doi: 10.1001/jama.2018.19320] [Medline: 30620375] 
42. Godfrey SS, Allender L, Laughery KR, Smith VL. Warning messages: will the consumer bother to look? Proc Hum Factors Ergon Soc Annu Meet 1983 Oct 01;27(11):950-954. [doi: 10.1177/154193128302701118]

43. Wogalter MS, Brelsford JW, Desaulniers DR, Laughery KR. Consumer product warnings: the role of hazard perception. J Safety Res 1991;22(2):71-82. [doi: 10.1016/0022-4375(91)90015-n]

44. Asimov I. I, Robot. London: Harper Voyager; 2013.

45. Asilomar AI principles. Future of Life Institute. 2017. URL: https://futureoflife.org/ai-principles/ [accessed 2021-09-30]

46. Parikh RB, Teeple S, Navathe AS. Addressing bias in artificial intelligence in health care. J Am Med Assoc 2019 Dec 24;322(24):2377-2378. [doi: 10.1001/jama.2019.18058] [Medline: $\underline{31755905]}$

47. Ermolina A, Tiberius V. Voice-controlled intelligent personal assistants in health care: International Delphi Study. J Med Internet Res 2021 Apr 09;23(4):e25312 [FREE Full text] [doi: 10.2196/25312] [Medline: 33835032]

48. Arrieta AB, Díaz-Rodríguez N, Del Ser J, Bennetot A, Tabik S, Barbado A, et al. Explainable Artificial Intelligence (XAI): concepts, taxonomies, opportunities and challenges toward responsible AI. Inf Fusion 2020 Jun;58:82-115. [doi: 10.1016/j.inffus.2019.12.012]

\title{
Abbreviations \\ AI: artificial intelligence \\ CA: conversational assistant \\ ECA: embodied conversational agent \\ TAM: Technology Acceptance Model
}

\author{
Edited by $G$ Eysenbach; submitted 26.05.21; peer-reviewed by V Tiberius, E Grekin; comments to author 17.08.21; revised version \\ received 29.08.21; accepted 18.09.21; published 09.11.21 \\ Please cite as: \\ Bickmore TW, Ólafsson S, O'Leary TK \\ Mitigating Patient and Consumer Safety Risks When Using Conversational Assistants for Medical Information: Exploratory Mixed \\ Methods Experiment \\ J Med Internet Res 2021;23(11):e30704 \\ URL: https://www.jmir.org/2021/11/e30704 \\ doi: $10.2196 / 30704$ \\ PMID:
}

(CTimothy W Bickmore, Stefán Ólafsson, Teresa K O'Leary. Originally published in the Journal of Medical Internet Research (https://www.jmir.org), 09.11.2021. This is an open-access article distributed under the terms of the Creative Commons Attribution License (https://creativecommons.org/licenses/by/4.0/), which permits unrestricted use, distribution, and reproduction in any medium, provided the original work, first published in the Journal of Medical Internet Research, is properly cited. The complete bibliographic information, a link to the original publication on https://www.jmir.org/, as well as this copyright and license information must be included. 